\title{
Familial hemiplegic migraine type i: the molecular signaling pathway
}

\begin{abstract}
Migraine is a multifactorial disease, manifested by intense bouts of recurrent headaches Molecular mechanisms of migraine attack are not clear. In this study, we carried out the analysis of molecular processes in the pathogenesis of a rare hereditary form of migraine familial hemiplegic migraine type I. Constructed hypothetical signaling pathways allowed us to understand the causes of a migraine attack and identify key molecules and signaling pathways for further experimental and clinical studies.
\end{abstract}

Keywords: familial hemiplegic migraine type I, fhm1, signaling pathways
Volume 7 Issue 5 - 2017

\author{
Eugene Klimov, 1,2 Olga Rudko, I Elena \\ Naumova,' Artemiy Tretiakov, ' Vladimir \\ Sobolev, ${ }^{2,5}$ Kirill Skorobogatykh, ${ }^{3}$ Julia \\ Azimova, ${ }^{3}$ Alexey Sergeev, ${ }^{3,4}$ Anna Soboleva, ${ }^{5}$ \\ Zarema Kokaeva, I Gyuzyal Tabeeva ${ }^{3,6}$ \\ 'Faculty of Biology of Lomonosov Moscow State University, \\ Russia \\ ${ }^{2}$ University diagnostic laboratory, Russia \\ ${ }^{3}$ University Headache Clinic, Russia \\ ${ }^{4}$ Department of Neuroscience, I.M.Sechenov First Moscow \\ State Medical University, Russia \\ ${ }^{5}$ Centre of Theoretical Problems of Physico-Chemical \\ Pharmacology, Russia \\ ${ }^{6}$ Department of neurology and neurosurgery, I.M.Sechenov First \\ Moscow State Medical University, Russia
}

Correspondence: Eugene Klimov, Department of Genetics, Biological Faculty of Lomonosov Moscow State University, 1 19234, Moscow, Lenin Hills, I-12, Russia,

Email klimov_eugeney@mail.ru

Received: August 21, 2017| Published: September 27, 2017

\section{Introduction}

Migraine is a multifactorial socially significant disease, manifested by intense bouts of recurrent pulsating headaches. There are three main theories describing migraine pathogenesis: vascular theory, neurogenic theory and trigeminovascular theory. Genetic predisposition to migraine is proved by epidemiological and genetic studies. Three forms of migraine with monogenic inheritance are well-known nowadays: familial hemiplegic migraine (FHM) of I, II and III types. But no genes or gene combinations associated with "common" migraine are known. Building a map of molecular signal pathways leading to a migraine attack may help to understand the causes of attacks and reveal key molecules and pathways. At the moment lack of information on genetic determinants of migraine and lack of animal models of "common" migraine makes it difficult to choose a starting point for a signal pathway. Because of this we have chosen FHM1 as a such point. FHM is a rare $(0.003 \%)$ and severe form of monogenic migraine with aura which is characterized by the development of muscle weakness during aura. It is considered that the mutations in CACNA1A gene which encodes the main subunit of voltage-dependent calcium channels (VDCCs) (Cav2.1) cause the development of FHM1.

Main function of VDCCs is modulation of stimulating neurotransmitters release in neuromuscular synapse as well as in central synapses of cerebellum, brain trunk and brain cortex predominantly. ${ }^{1}$ More than 60 mutations which may lead to different phenotypical forms varying from pure FHM1 to FHM1 accompanied by cerebellar ataxia of varying severity or severe brain edema leading to fatal coma are known nowadays in this gene..$^{2-4}$ Mutations in CACNA1A gene may cause diseases not linked to FHM1 including episodic ataxia type $2,{ }^{5}$ progressive ataxia, ${ }^{6}$ spinocerebellar ataxia type $6^{7}$ and different forms of epilepsy. ${ }^{8}$ The development of FHM1 is mainly caused by missense mutations in CACNA1A gene (50-70\% of families). ${ }^{9}$ In $40 \%$ of families with FHM1, a mutation leading to Thr666Met amino acid substitution was found (here and further the location of a substitution with a number between two amino acid names was marked). ${ }^{10}$ Arg $192 \mathrm{Gln}$ mutation causes pure form of hemiplegic migraine, while Ser218Leu causes malignant form with the hemiplegic migraine attacks developing after any head injury and often accompanied by coma. ${ }^{11}$ Meanwhile Arg192Gln homozygotes may show normal phenotype, Ser218Leu heterozygotes are characterized by ataxia and high risk of sudden death due to severe epileptic seizures and cerebral edema. ${ }^{11,12}$ It was shown on the cell models that different types of mutations of CACNA1A gene in FHM1 cause different variants of channelopathies - a type of diseases characterized by ion channel function disturbance, changes in its structure and kinetics, ${ }^{13-16}$ leading to increased $\mathrm{Ca} 2+$ ion flow through voltage-dependent channels. Altered calcium channels may be opened by less voltage comparing to wild-type channels which means that less depolarization is required to open a channel. ${ }^{11}$ Moreover, altered channels are opened for a longer time compared to wild type. Mutation Ser218Leu results in the development of the most altered channels and shows the most severe phenotype with risk of severe attacks accompanied by consciousness failures. ${ }^{17}$

Purpose of work - conduct analysis of molecular processes underlying pathogenesis of familial hemiplegic migraine type I. Building schemes of molecular interactions which lead to migraine attack development will allow not only to understand causes of an attack but also to reveal key molecules and pathways which may be targets for new drugs and treatment approaches. 


\section{Materials and methods}

In our study, we used Pathway Studio ${ }^{\circledR} 9$ software with ResNet $\AA$ 13 (Elsevier) database. ResNet13 contains biological objects (proteins, cell processes and diseases in particular) with annotations, as well as annotations of function links between objects. This database is a result of processing full-text research papers and abstracts indexed by Medline.

\section{Results and discussion}

We created a hypothetical scheme of signal pathways describing causes and possible mechanisms of aura, vasodilation and pain in FHM1. The scheme is shown on figure 1, CACNA1A is marked white (Figure 1). Cav2.1 channel consists of four proteins CACNG2, CACNA2D2, CACNB4 and CACNA1A. Cav2.1 main function is modulation of dominantly excitatory neurotransmitter release in neuromuscular synapses as well as in central synapses of cerebellum, trunk and cortex. ${ }^{18}$

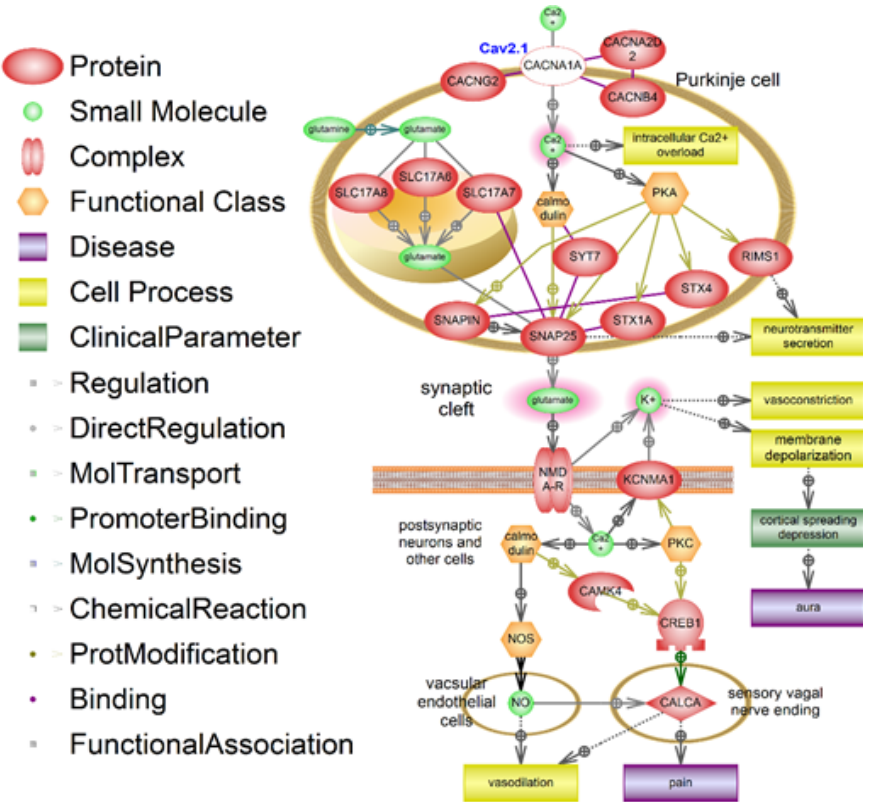

Figure I Signaling pathway of familial hemiplegic migraine type I (FHMI). Mutations in CACNAIA (white-out style) lead to intracellular calcium overload, glutamate overdose in synaptic cleft, development of spreading cortical depression (SCD), migraine aura, vasodilation and pain. Molecules with increased concentrations are highlighted in red. Legend is shown on the Figure. Designed in the Pathway Studio $9 \circledR$ (Elsevier). This signaling pathway is built manually using Res Net I 3 database $\AA$ (Elsevier).

In Purkinje cell 3 vesicular glutamate transporters - SLC17A7, SLC17A6 and SLC17A8 - transport synthetized glutamate to vesicles. Glutamine is being synthetized in astrocytes and transported to neurons. The fusion of vesicles with presynaptic membrane is initiated by intercellular calcium by activation of calmodulin and protein kinase A (PKA). Calmodulin and PKA phosphorylate activate main proteins of exocytosis such as SNAP25, SNAPIN, SYT7, STX1A, STX4 and RIMS1, which take part in neurotransmitter secretion.

Thus, CACNA1A dysfunction leads to uncontrollable calcium intake which leads to recurrent glutamate release to the synaptic cleft. This is supported by clinical data since glutamate release inhibitor (botulin toxin A) alleviates the migraine symptoms. Main target of botulin toxin A is SNAP25 a protein which plays lead role in fusion of vesicles containing neuromediators with cell membrane. Thus, the pathological increase of glutamate concentration in synaptic cleft is a key part of our scheme.

Increased glutamate release activates NMDA receptors on postsynaptic neurons which lead to release of intracellular potassium to cell surface and calcium intake. Extracellular potassium causes depolarization of membrane. Hyperpolarization is the main cause of spreading cortical depression characterized by spreading polarization of brain cells. Aura antecedent to migraine attack is the result of spreading cortical depression. Recent studies showed that the increase of glutamate concentration plays a leading role in the development of spreading cortical depression supporting the hypothesis of cortical hyperexcitability in migraine. ${ }^{19}$

Potassium release leads to vasoconstriction of nearby vessels. Vasoconstriction appears before vasodilatation and occurs in parallel with development of spreading cortical depression. Intracellular calcium in postsynaptic neurons activates KCNMA1 channel, leading to lengthening of depolarization. Intracellular calcium activates cell specific (neuronal or vascular-endothelian) types of NO-synthases (NOS) via calmodulin which results in increased synthesis of nitric oxide (NO) - a strong vasodilator and CGRP (CALCA) release activator. Synthesis and release of the main vasodilator and pain neuromediator CGRP (CALCA) in sensory vagus nerve ending is also activated. This neuropeptide causes the main pathological processes appearing in migraine attack: vasodilation and pain.

\section{Conclusion}

Thus we are the first to create a model of signal pathways leading to FHM type I. The key molecular in this model is synaptic glutamate. And major processes are intracellular calcium overload in Purkinje cell and membrane depolarization after glutamate overdose. This model may be used as a starting point for identification of further pathways of "common" migraine.

\section{Disclosures}

\section{Acknowledgements}

This research received no specific grant from any funding agency in the public, commercial, or not-for-profit sectors.

\section{Conflict of Interest Statement}

No conflict.

\section{Author's contributions}

Conception: Eugene Klimov, Olga Rudko, Julia Azimova, Gyusal Tabeeva

Literature Analyses: Elena Naumova, Julia Azimova, Alexey Sergeev, Kirill Skorobogatykh, Anna Soboleva, Zarema Kokaeva

Pathway Design: Eugene Klimov, Artemiy Tretiakov, Vladimir Sobolev

Manuscript Preparation: Eugene Klimov, Artemiy Tretiakov

Writing of the first draft: Eugene Klimov, Olga Rudko.

\section{Acknowledgments}

None.

\section{Conflicts of interest}

None. 


\section{Funding}

None.

\section{References}

1. Thomsen L, Ostergaard E, Romer SF, et al. Sporadic hemiplegic migraine is an aetiologically heterogeneous disorder. Cephalalgia. 2003;23(9):921-928.

2. Catterall WA. Structure and function of neuronal $\mathrm{Ca} 2+$ channels and their role in neurotransmitter release. Cell Calcium. 1998;24(5-6):307-323.

3. De Vries B, Freilinger T, Vanmolkot KR, et al. Systematic analysis of three FHM genes in 39 sporadic patients with hemiplegic migraine. Neurology. 2007;69(23):2170-2176.

4. Rajakulendran S, Kaski D, Hanna MG. Neuronal P/Q-type calcium channel dysfunction in inherited disorders of the CNS. Nat Rev Neurol. 2012;8(2):86-96.

5. Ophoff RA, Terwindt GM, Vergouwe MN, et al. Familial hemiplegic migraine and episodic ataxia type- 2 are caused by mutations in the $\mathrm{Ca} 2+$ channel gene CACNL1A4. Cell. 1996;87(3):543-552.

6. Yue Q, Jen JC, Nelson SF, et al. Progressive ataxia due to a missense mutation in a calcium-channel gene. Am J Hum Genet. 1997;61(5):1078-1087.

7. Zhuchenko O, Bailey J, Donnen P, et al. Autosomal dominant cerebellar ataxia (SCA6) associated with small polyglutamine expansions in the a1A-voltage-dependent calcium channel. Nature Genetics. 1997;15:62-69.

8. Jouvenceau A, Eunson LH, Spauschus A, et al. Human epilepsy associated with dysfunction of the brain P/Q-type calcium channel. Lancet. 2001;358(9284):801-807.

9. De Vries B, Haan J, Frants RR, et al. Genetic biomarkers for migraine. Headache. 2006;46(7):1059-1068.
10. Ducros A, Diener C, Joutel A, et al. Recurrence of the T666M calcium channel CACNA1A gene mutation in familial hemiplegic migraine. Am J Hum Genet. 1999;64(1):89-98.

11. Pietrobon D. Biological science of headache channels. Handb Clin Neurol. 2010;97:73-83.

12. van Den Maagdenberg A, Terwindt G, Haas J, et al. Genetics of headaches. Handb Clin Neurol. 2010;97:85-97.

13. Cao YQ, Piedras-Renteria ES, Smith GB, et al. Presynaptic Ca2p channels compete for channel type-preferring slots in altered neurotransmission arising from Ca2p channelopathy. Neuron. 2004;43(3):387-400.

14. Hans M, Luvisetto S, Williams ME, et al. Functional consequences of mutations in the human alpha(1A) calcium channel subunit linked to familial hemiplegic migraine. J Neurosci. 1999; 19(5):1610-1619.

15. Kraus RL, Sinnegger MJ, Koschak A, et al. Three new familial hemiplegic migraine mutants affect $\mathrm{P} / \mathrm{Q}$-type $\mathrm{Ca}(2 \mathrm{p})$ channel kinetics. $J$ Biol Chem. 2000;275(13):9239-9243.

16. Tottene A, Tottene A, Fellin T, et al. Familial hemiplegic migraine mutations increase $\mathrm{Ca} 2+$ influx through single human cav2.1 channels and decrease maximal cav2.1 current density in neurons. Proc Natl Acad Sci U S A. 2002;99(20):13284-13289.

17. Stam AH, Louter MA, Haan J, et al. A long-term follow-up study of 18 patients with sporadic hemiplegic migraine. Cephalalgia. 2011;31(2):199-205.

18. Cao YQ, Piedras Renteria ES, Smith GB, et al. Presynaptic Ca2+ channels compete for channel type-preferring slots in altered neurotransmission arising from Ca2+ channelopathy. Neuron. 2004;43(5):387-400.

19. Zielman R, Wijnen JP, Webb A, et al. Cortical glutamate in migraine. Brain. 2017;140(7):1859-1871. 